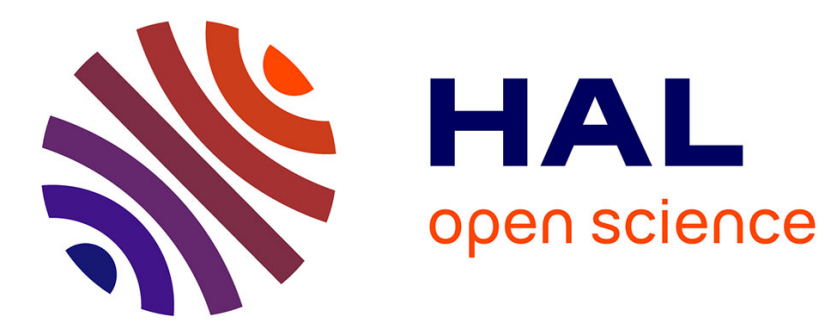

\title{
Parametric point cloud slicing for facade retrofiting
}

Oscar Gámez Bohórquez, William Derigent, Hind Bril El-Haouzi

\section{To cite this version:}

Oscar Gámez Bohórquez, William Derigent, Hind Bril El-Haouzi. Parametric point cloud slicing for facade retrofiting. International Journal of Architectural Computing, In press, $10.1177 / 14780771211029747$. hal-03276172

\section{HAL Id: hal-03276172 \\ https://hal.science/hal-03276172}

Submitted on 1 Jul 2021

HAL is a multi-disciplinary open access archive for the deposit and dissemination of scientific research documents, whether they are published or not. The documents may come from teaching and research institutions in France or abroad, or from public or private research centers.
L'archive ouverte pluridisciplinaire HAL, est destinée au dépôt et à la diffusion de documents scientifiques de niveau recherche, publiés ou non, émanant des établissements d'enseignement et de recherche français ou étrangers, des laboratoires publics ou privés. 


\title{
PARAMETRIC POINT CLOUD SLICING FOR FACADE RETROFITTING
}

\author{
Oscar Gámez¹, William Derigent, Hind Bril El houzi \\ Université de Lorraine, CRAN CNRS UMR 7039, 88000, Epinal, France.
}

\begin{abstract}
Current commitments by European governments seek to improve energy consumption as a means to reduce carbon emissions from building stock by 2050 . Within such context, retrieving reliable threedimensional contours from point clouds becomes an important step in developing facade retrofitting solutions since facade retrofitting projects often make use of as-built 3D models to help reduce inaccuracies by narrowing interpretation and measurement errors. This work aims to provide a method that uses topology-based parametric modelling for reconstructing building envelopes from point clouds. Through a semi-automated process that gives permanent visual feedback, the user adjusts parameters to custom standards of acceptability. A solution under the form of a Grasshopper definition delivers building envelope 3D contours in various file formats as a means for increasing interoperability. The main contributions of this work consist of a parametric reconstruction workflow capable of solving building topology for retrieving 3D contours, a strategy to bypass point cloud occlusion, and a strategy for converting those contours into an IFC model directly from the parametric modelling environment.
\end{abstract}

\section{Keywords}

Facade retrofitting, Scan to BIM, Parametric modelling, Terrestrial laser scanning, Building life cycle.

\section{Introduction}

As the use of Building Information Modelling for the Architecture, Engineering, Construction and Owner-operated (AECO) industry becomes widespread, its full potential is achievable by its implementation throughout the entire lifecycle of buildings. Within such scope, the need for as-built information becomes relevant inasmuch as current commitments by European governments aim to improve energy consumption as a means to reduce carbon emissions, which in turn, are expected to help control climate change by decarbonizing the European building stock by 2050 [1]. This is mainly because at least $75 \%$ of the European building stock is energy inefficient[2], which reveals the need for adopting retrofitting strategies aimed at accomplishing energy consumption goals.

As-built information in the form of object modelling has proved to be relevant to the AECO industry in the framework of Operations and Maintenance (O\&M), Facilities Management (FM) and building retrofitting[3]. In order to provide the AECO industry with reliable information, data acquisition techniques based on point clouds obtained using terrestrial laser scanning (TLS) provide a starting point for structuring solid building data[4]. As-built 3D models, constructed from point clouds (hereafter PC_Sets), reduce inaccuracies by narrowing interpretation which is a common problem when working with unstructured 2D as-built documentation[5]. Furthermore, retrieving reliable contours from PC_Sets helps develop nesting solutions for facade retrofitting by providing accurate

\footnotetext{
${ }^{1}$ Corresponding author. E-mail address: gamezboh1@univ-lorraine.fr (Oscar Gámez). ORCid 00000003-3973-3343
} 
references of levels, locations, paths and dimensions for key building envelope components such as walls, slabs, columns, doors and windows.

The literature cited throughout this paper, which spans seven years, shows that the last four years have seen an increasing activity in the study of the aforementioned topics (figure 1). Many of them have addressed the problem of converting point clouds into BIM models by utilizing image treatment and PC_Set segmentation techniques, whereas some others have proposed geometrically based methods to generate as-built contours. However, very few have considered the possibility of using a native modelling environment to do so.

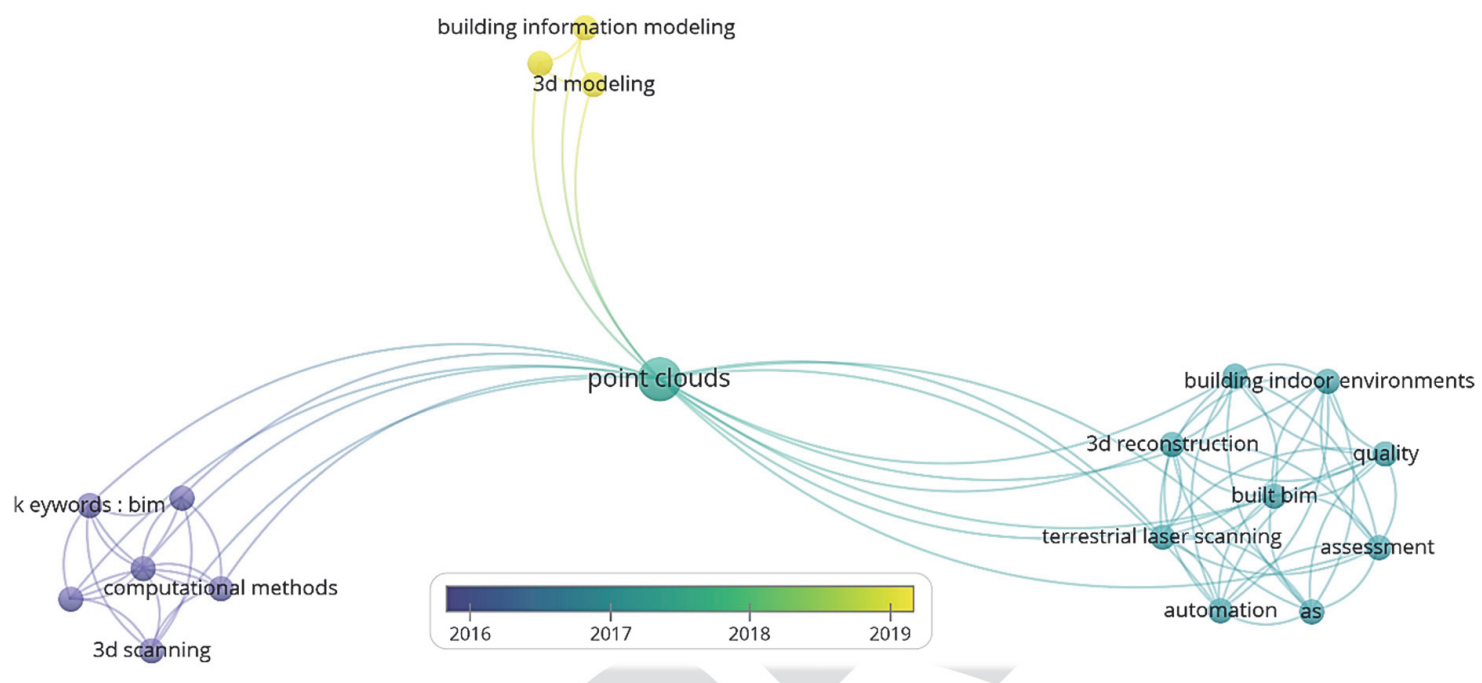

Figure 1. Keyword map of recent works dealing with building reconstruction from point clouds

This paper investigates an alternative for digitally reconstructing building contours for facade retrofitting by proposing a digital method for as-built contour reconstruction using a native Nurbs ${ }^{2}$ modeler as akin endeavors by computing engineers often employ tools not easily accessible or understandable to designers.

By using Nurbs modelling through visual programming and algorithmic design[6, 7], whose utilization among practitioners and researchers in architecture and design is well known[8,9], this work proposes a PC_Set-slicing method for acquiring as-built contours usable for facade retrofitting projects. The aforementioned method constitutes the main contribution of this work.

The obtained results are compatible with facade retrofitting projects such as those of Borodinecs et al, Barco et al. or Gámez et al. [10-12] for which 3D boundary representations help start exterior insulation nesting endeavors whose insulation pattern plays an aesthetic role.

The remainder of this paper is structured as follows:

Section 2 identifies the gaps aimed to be tackled within the context of this paper by focusing on related literature, followed by a description of the goals to reach in Section Three. Section Four describes the methodology for fulfilling those goals while simultaneously depicting the general architecture of the proposed Grasshopper solution $(\mathrm{GH})$ used for retrieving 3D building envelope boundaries from point clouds, along with a detailed description of the sub-processes composing it.

To show one of the possible ways in which the digital continuum proposed here can serve a purpose, a test, which consists of converting 3D building boundaries into IFC entities by using an IFC plug-in for $\mathrm{GH}$, is presented along with is results. By the end of the paper we present the general results and state this work's conclusions and its perspectives for future research.

\footnotetext{
${ }^{2}$ Non-uniform B-Spline
} 


\section{Related work}

In recent years, methods and strategies for retrieving as-built information from PC_Sets utilizing image processing and mathematical procedures show an important progress in the field, since efforts focus on retrieving detailed information useful for creating as-built BIM models suitable for building retrofitting, Facilities Management (FM), Heritage management (HM), and Asset Management (AM) $[10,13-15]$. As some works focus on item recognition from images[16], which still have to overcome accuracy issues[17], others concentrate on solving more specific problems such as complex spaces[18, 19] or facade arrangements[4]. Other than the focus, challenges arise when recognizing elements such as doors or windows, processing facade patterns, or when resolving contour distortions caused by occluding objects and/or topologic variations.

A study by Previtali [20] outlines the importance of resolving occlusion issues affecting reconstruction quality while at the same time tackling basic semantics aimed at recovering data to characterize items like doors, windows, walls, floors and ceilings. It also deals with undesired occlusion effects when reconstructing geometries from point clouds, which is a recurrent topic. Jung[21], for instance, acknowledges that voids resulting from occlusion are often smaller than actual window or door openings; a problem that Macher [19] solved by automating the differentiation between occlusionoriginated voids and actual openings and then filling the voids with point cloud patches. The work of Wang[16] acknowledges that such occlusion, being a point cloud source problem, sets limitations to the outcome of his research. The tool proposed by Abdelhamid [22] processes PC_Sets by means of Ransac and Hough algorithms that retrieve wall, window and door contours grouped as collection of exportable 3D-DXF vectors usable in BIM modelers as paths for walls as well as location references for windows and doors. Despite its potential, occlusion issues are unresolved because occluded surfaces turn into voids that usually merge with window and door contours. The works by Hackel, Xue, Adan and Krop [4, 23-25] also mention strategies regarding the challenges generated from occlusion in automated or semi-automated building reconstruction from point clouds.

Throughout the referred works, retrieving building geometry from laser scan data in the form of dense and regular sample points[26] involves using the Random Sample Consensus (RANSAC)[27], or Hough, Roberts, Canny or Laplacian algorithms $[16,22,28]$. Other methods, however, appear to tackle similar problems by entailing alternative approaches. Bassier et al. [29], for instance, propose a "Conditional Random Field" that evaluates the context of each building's wall in order to determine its position among complex spatial configurations. Laing [30] uses Poisson algorithms to turn pointcloud data into uniform meshes, which are later sliced in order to obtain contour data usable in the CNC machining of mockups. Meshes serve then as a bridge for retrieving geometric building data.

With an akin goal - and making use of topologic relations - the work of Huhnt [31] addresses the reconstruction of contours from mesh-generated representations, which are later subdivided into triangle sets and edges suitable for creating three-dimensional representations of building components at low LOD's[32].

The trend gradually goes from pure point cloud processing to mesh modelling and into parametric Nurbs modelling as strategies for rebuilding geometry from PC_Sets. López et at. [33] pinpoint at least five works that use Nurbs modelling as a resource for retrieving point cloud cross sections later used in the parametric model aimed at reconstructing the scanned entity. Although at least three of those works claim to make use of the Rhinoceros environment to perform part of the process, they do not disclose detailed processes as proof.

A procedure developed by Barazzetti[34] slices point clouds in order to retrieve several cross-section Nurbs curves that later help in the modelling of complex vaults as point cloud profiles are manually extracted for further processing via an automated NURBS surface[35] generator[36]. Its results show that having several PC_Set slices facilitate the retrieval of topological variations that result in increased reconstruction accuracy.

Following the same trend, the work of Dimitrov[37] also uses point cloud slicing as a method to produce cross-section lines later used in reconstructing building geometries; this strategy tackles the 
building as a whole and does not provide a mechanism for treating parts of it in an isolated manner. As the method is developed within Matlab, compatibility or expandability towards disciplines other than computing engineering is not provided.

The previously mentioned methods and studies remain close to the goals this work seeks to reach, which demonstrates that the topic is of interest and in constant evolution. It also shows that research endeavors cover aspects not usually developed by industrial solutions like Faro As-built[38], Scan to BIM[39], Scalypso[40] or Leica Cloudworx[41]. These industrial solutions offer reliable tools for most standard reconstruction cases yet they do not provide accurate solutions for treating topologic variation as they rely on standard modeling capabilities from native BIM modelers. This is where this work seeks to make a contribution to the field.

\section{Research aim}

Point cloud deconstruction and segmentation has been widely explored in recent years since the use of PC_Sets for architectural surveying has become commonplace in mid-size and large renovation and retrofitting projects $[10,11]$. This work aims to provide a point cloud-based surveying method utilizing the Rhinoceros-grasshopper environment (RGH). This method uses a topology-based tool for retrieving essential contours for reconstructing retrofitting-suitable building envelopes.

The parametric modelling workflow[42] utilizes RGH along with plug-ins and custom scripts whose purpose is to deliver the three-dimensional contour of a given building. To validate the approach, we conducted a test on a building whose stories do not share coplanar facades thus facade planes are not continuous, which is a problem not often explored in the digital reconstruction of building envelopes. Furthermore, the three-dimensional outcome as a common file format is thought to be interoperable with specialized software for exterior insulation simulation and nesting. To this extent, all the user needs to process PC_Sets is a point cloud editor such as Autodesk Recap Pro or Cloud Compare and a recent version of $\mathrm{RGH}$.

As for data including the building's structure, the amount of retrieved geometry depends on how concealed structural elements are. Structural concealment is more frequent in framed structural systems than in wall-bearing systems, although this fact is not a constant.

\section{Methodology}

As the purpose focuses on the extraction of three-dimensional contours of building-envelopes from unstructured PC_Sets, point clouds are treated in the same manner as they would within BIM modelers such as Revit, Archicad or AllPlan.

The system is composed by thirteen sub-processes driven by adjustable parameters the user gradually evaluates as calculations take place within the RGH environment (Table 1). Through a semi-automated process that gives permanent visual feedback, the user adjusts parameters to custom standards of acceptability. A solution under the form of a Grasshopper definition [43] delivers building envelope 3D contours as a DWG, 3DM, 3DS or DXF files.

\subsection{Workflow.}

As shown in Figure 2, the process starts by importing a PC_Set fetched as an E57 file whose density can be reduced in order to improve system performance, since in further stages the PC_Set undergoes several transformations into voxel sets (VoxSet).

As PC_Sets often have their own coordinate systems, the solution provides a mechanism that places the point cloud onto a locally defined coordinate system, which is achieved by translating the point cloud to a reference plane defined by the user (Table $1, \# 1$ ). 


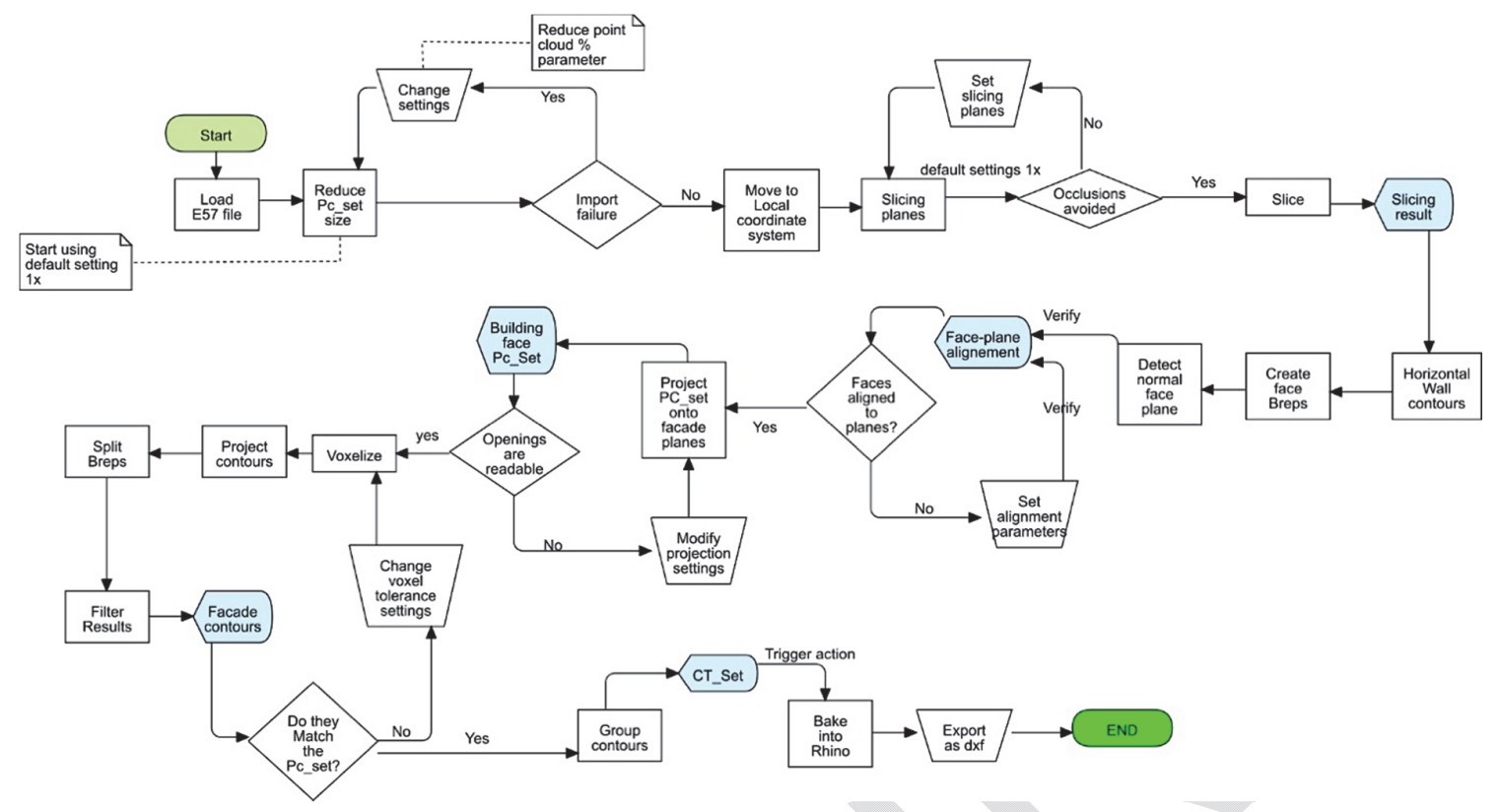

Figure 2. General method and GH definition workflow

The PC_Set is then intersected by a set of slicing planes (Sp_Set) that produces cross-section contours[44] or contour sets (Ct_Set), which we will use later for reconstructing each of the planes parallel to every building's face (F_C_Plane) (Figure 3 ). As occlusion appears to be an important issue when reconstructing building geometries out of point clouds $[20,21,44]$, the user can use a multipleentry slider in the RGH definition to adjust the relative height of each plane composing the Sp_Set to skip occluded regions (Table $1, \# 3$ ).

Because occlusion mostly occurs at levels close to the ground, the possibility of having missing PC_Set segments might induce an error when detecting footprints of envelope walls. In such case, the Sp_Set computes a vector ( $\mathrm{Sp} p_{-} v$ ) by connecting origin points from each plane composing the $S p_{-}$Set, which in turn will project a substitute footprint for walls affected by occlusion (Table 1 \#7).
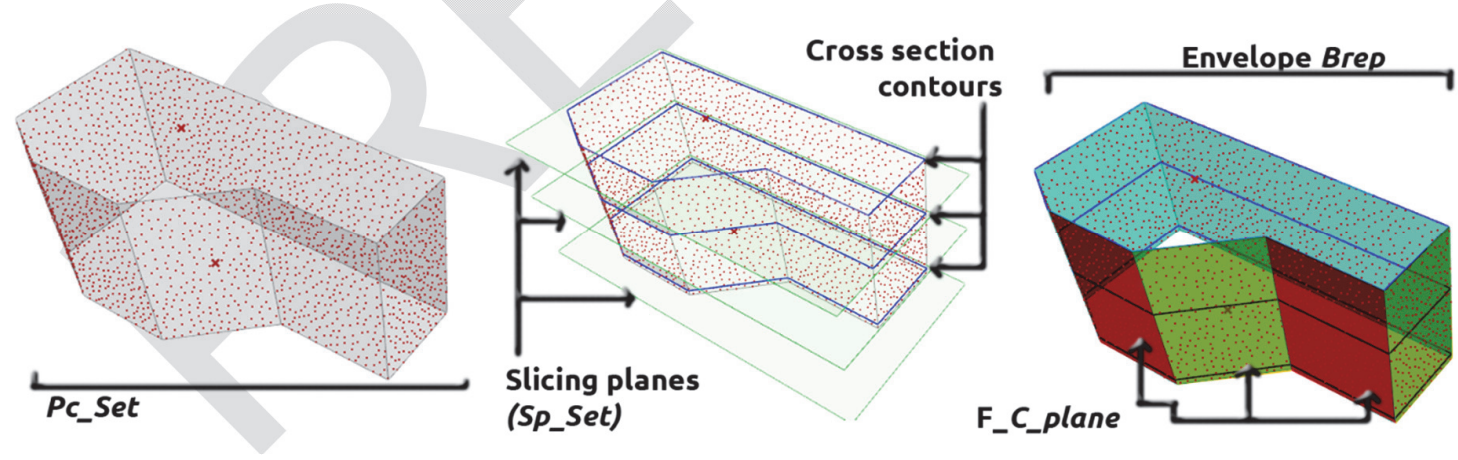

Figure 3. LEFT, Point cloud set (PC_Set); CENTER, Slicing planes and Pc_Set cross-sections. RIGHT, Envelope Brep after reconstruction and building face construction planes (F-C_Plane)

Unlike wall footprints, retrieving boundaries for windows and doors is achieved by projecting segments of the PC_Set parallel to the corresponding facade construction plane (F-C_Plane). After facade planes are detected, a $1^{\text {st }}$ degree Nurbs Surface [35] containing face boundaries $\left(B r e p s^{3}\right)$ is created using the F_C-Plane as reference. The user uses the same F_C-Plane as an attractor to project a certain quantity of points from the PC_Set onto the Brep, so openings are clearly readable on the

\footnotetext{
${ }^{3}$ Boundary representations in the form of Nurbs Surfaces are noted as Breps.
} 
screen. After a voxelization process, a boundary polyline - which is later refined to cull undesired vertexes to smoothen resulting contours- is created for each building's face (Table 1, \#8).

The $\mathrm{GH}$ solution uses openings contours to split the facade Brep in order to obtain a geometric 3D representation of building facades (as Breps). Constituents of such Breps are then extracted to retrieve final contours, group them and then bake them into Rhinoceros prior to exporting them as a 3D polyline set, which is the final output (Table 1, \#9). Section 4.2 describes in further detail each of the sub processes of the RGH definition.

\subsection{Sub process description}

Table 1. List of sub-processes within the GH definition.

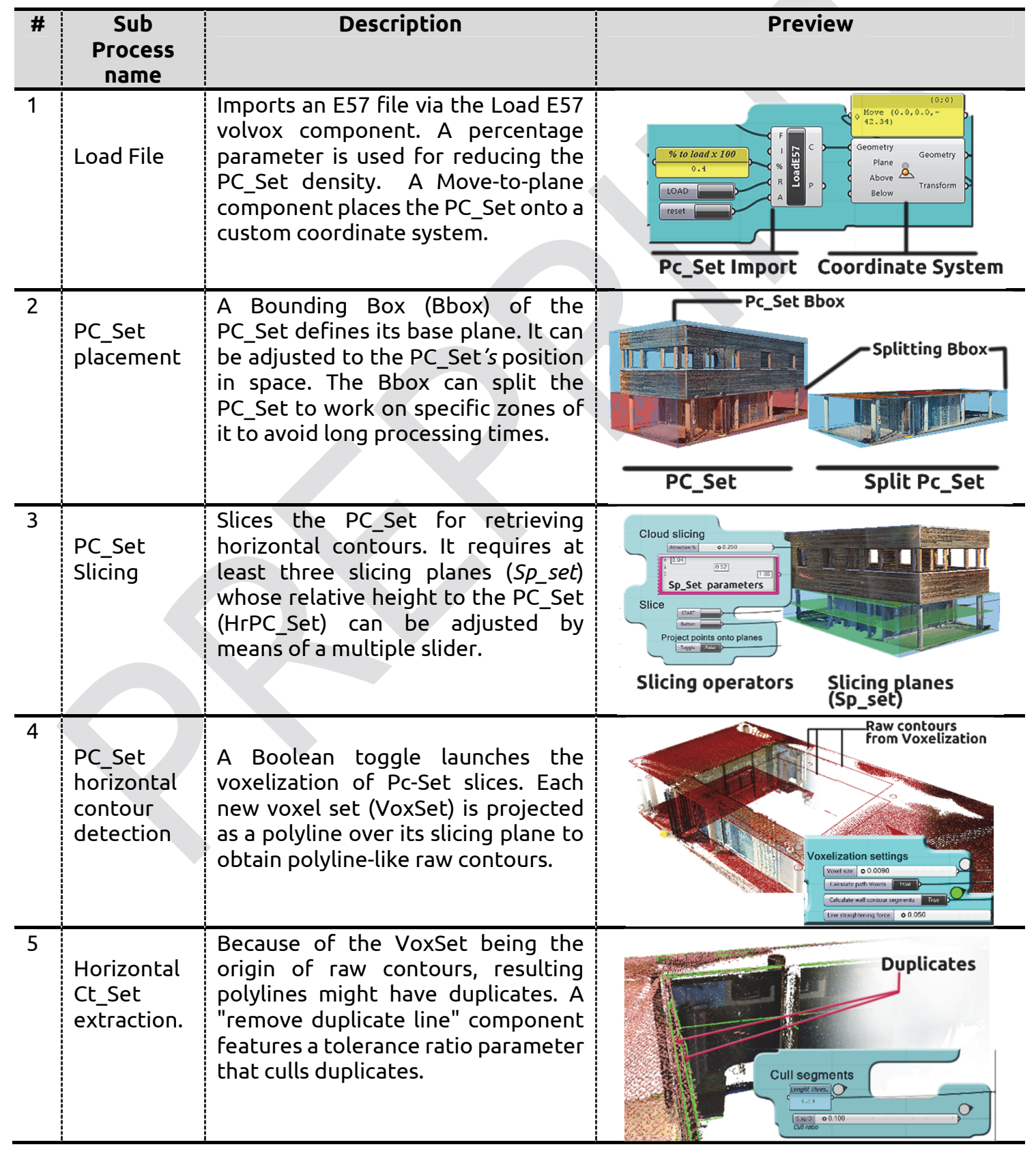




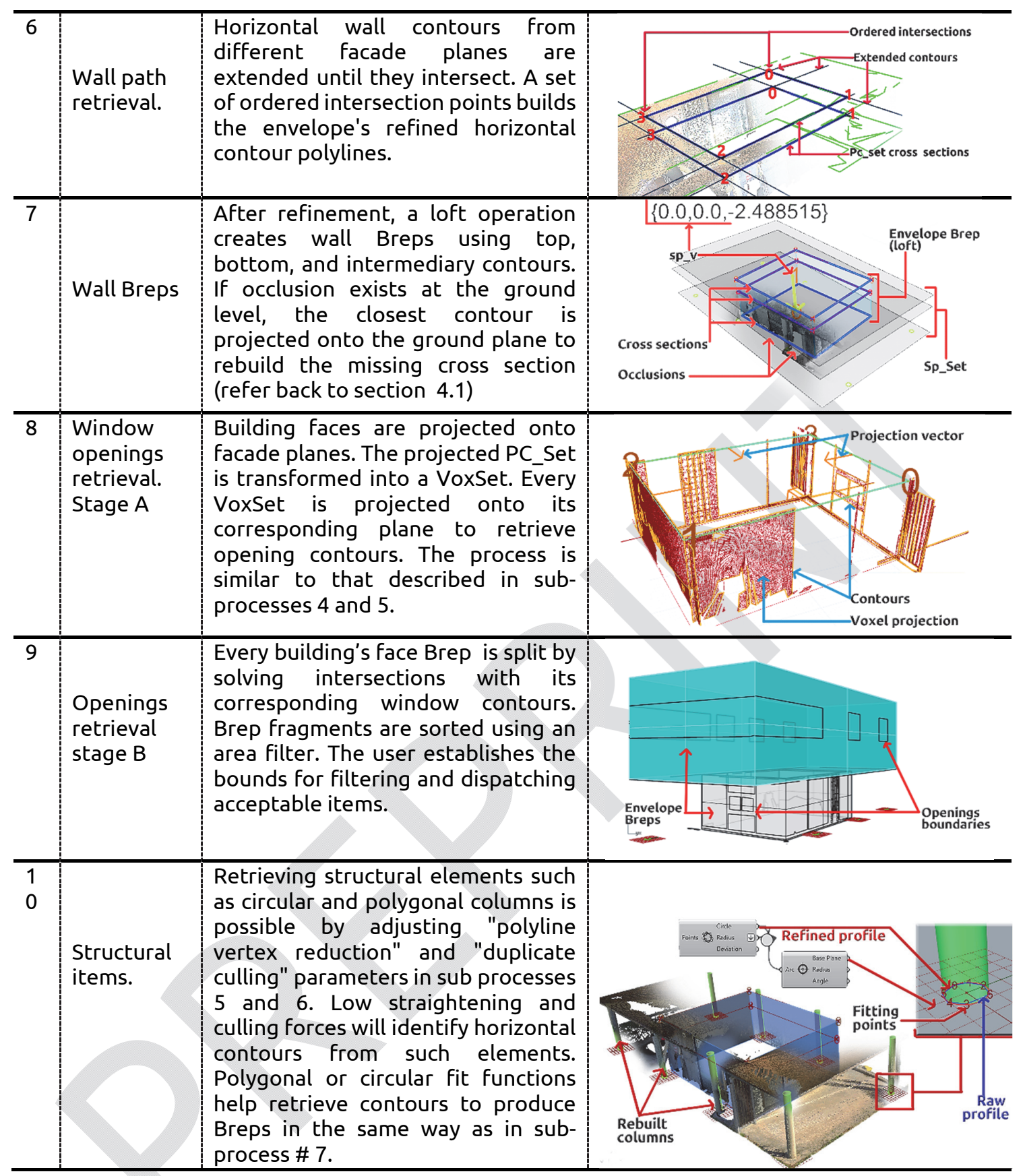

The results shown so far are the product of testing the GH solution against a complete PC_Set. Partial tests with other point clouds were performed in order to refine the solution's parameters so that when performing the tests it would be possible to measure the time it takes to process a given point cloud. The PC_Set used for testing the GH solution had about 12.8 million points that were reduced to 5.13 million before running the $\mathrm{GH}$ algorithm.

The observations show that with a PC_Set that size, most processes react within a time range between $200 \mathrm{~ms}$ to $400 \mathrm{~ms}$, although there is an exception. Calculating contours from Voxel projections takes up to 2 minutes when applied to the second floor and up to 4.5 minutes when used for the first floor. This is mainly because of higher point cloud noise in the first-floor segment. 
The aforementioned process delivers a three-dimensional Contour set (Ct_Set) representing the building's envelope. In order to set a foreseeable use scenario, the obtained contours were transformed into IFC entities directly within RGH. The process is depicted in the next section.

\subsection{BIM compatibility}

As there is an increasing use of Building Information Modelling (BIM) in the AECO industry, the IFC standard offers several manners for converting simple 3D representations into interoperable files. Such is the case of the RGH environment for which various BIM-aimed add-ons such as Grevit[45], GeomGym IFC[46] or VisualArq [47], which help translate architectural shapes into IFC compatible models, have been under development. In more recent developments of Autodesk Revit, it is possible to run RGH "within Revit's memory space"[48], thus increasing interoperability and workflow flexibility.

Although BIM integration does not constitute the main goal of this work, providing an overview about how the results shown herein are translatable into IFC data might help understand the usefulness of this approach.

A test using DURAARK's plug-in for RGH, GeomGym IFC, was performed. The plugin helped in creating parametric IFC entities that are later suitable for exporting as a $2 \times 3$ IFC file. While GeomGym IFC provides the necessary tools for creating building components following the levels of decomposition defined by the IFCSpatialstructureElements supertype [49,50], the 3D building contours obtained using our method provide an accurate basis for generating building geometry. This is possible because GeomGym IFC mainly requires lines and reference points for creating IFC entities (Figure 4, left).

3D contours were decomposed into their basic elements using standard $\mathrm{GH}$ components, which in turn allowed the retrieval of building data in the form of reference planes, building and story heights and placements, dimensions and positions for openings, and walls and columns (Figure 4, Left-Right).

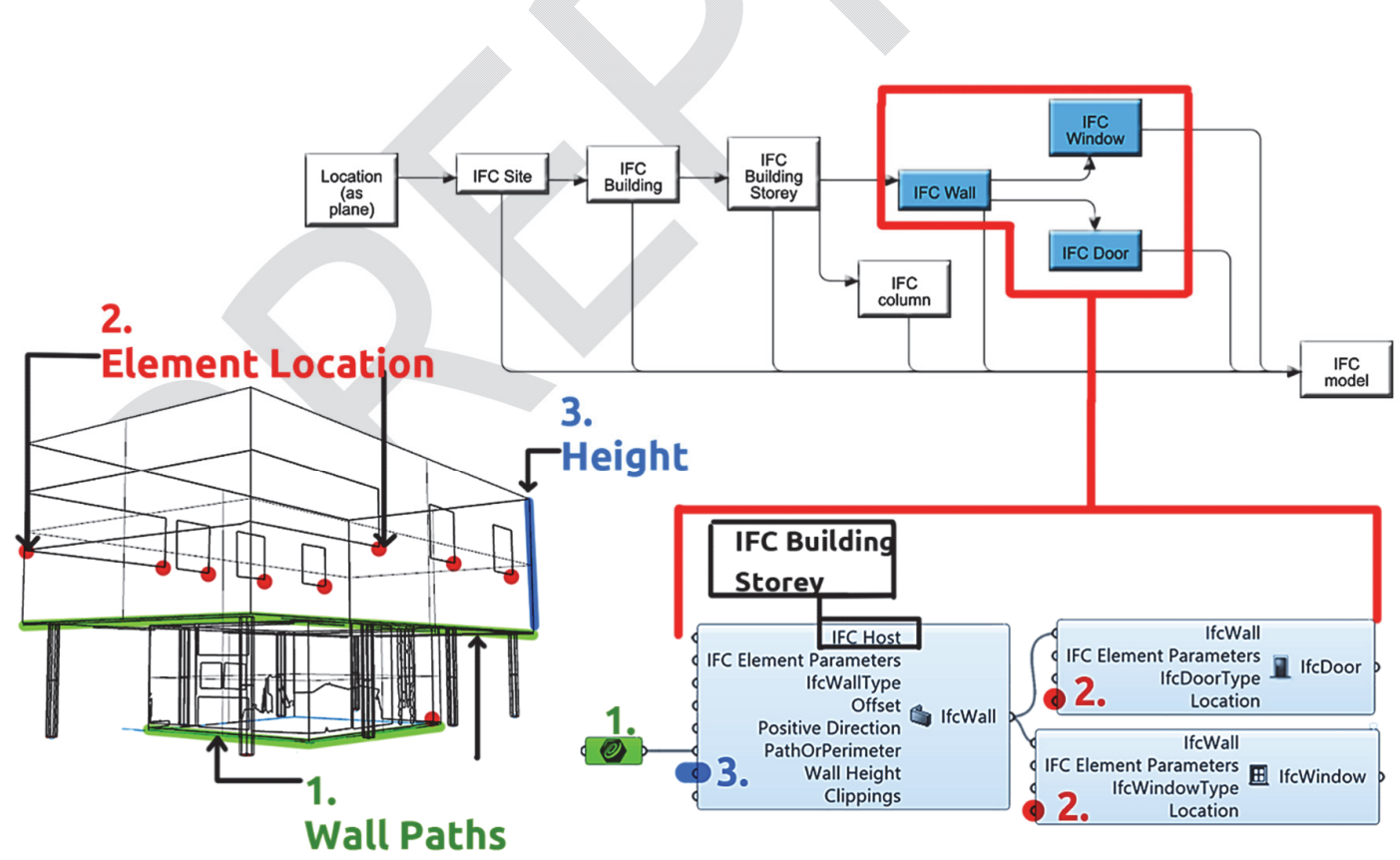

Figure 4. IFC model generation from 3D contours. UP, Model hierarchy. LEFT, 3D Building contours. RIGHT, IFC elements within GeomGym IFC.

Notice that despite the fact the graphical representation looks acceptable, erroneous IFCElemenParams are not graphically displayed, therefore, an erroneous model is not visible. Usually, IFCElemenParams errors are detected after exporting the file and opening it with an IFC viewer or 
native BIM program, in which case there will be missing elements, disaggregated entities or no entities at all. It is advised to check IFCElemenParams before exporting them to detect errors. For more information about the correct utilization of GeomGym IFC, we encourage the reader to test GeomGym IFC following the supporting documentation provided by its developer.

The performed test yielded a LOD 100 model whose IFC file reads well in Revit and Archicad for further enrichment (Figure 5).
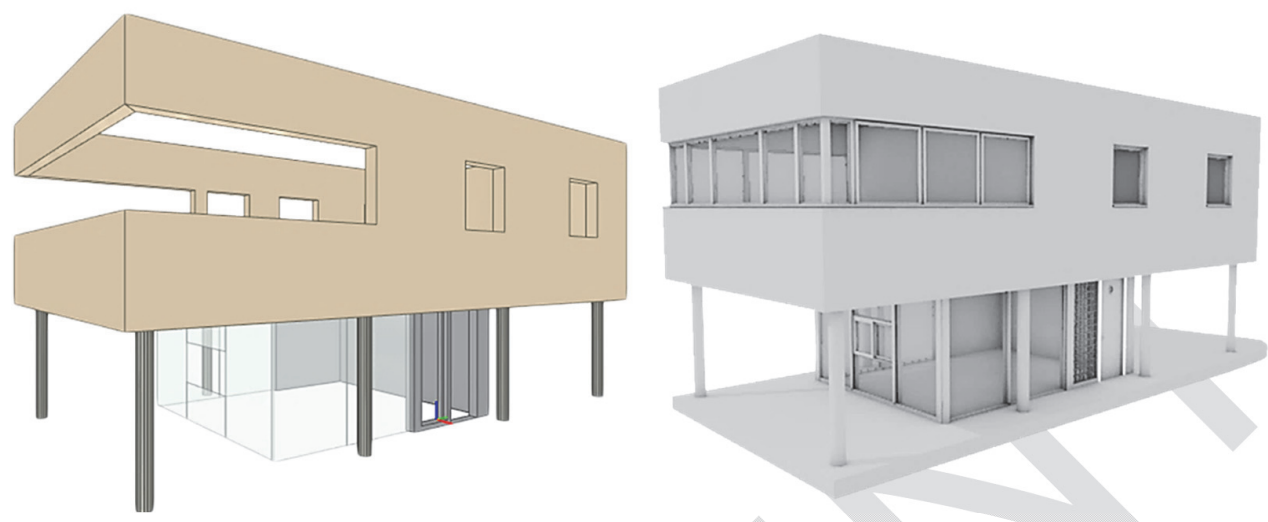

Figure 5. LEFT, 3D model as obtained from RGH. RIGHT. Enriched model using Archicad

\section{Results}

The achieved results show that the method, along with its RGH, definition can produce threedimensional Ct_Sets from point clouds, which are usable in facade retrofitting projects (Figure 6). By deconstructing PC_Sets using parametric Nurbs modelling, it is possible for the user to have a feedback from the solution and progressively adjust its settings as needed.

The modules within the $\mathrm{GH}$ definition were tested in the reconstruction of a building's envelope whose stories do not possess co-planar facades and whose ground floor facades are affected by random occlusions.

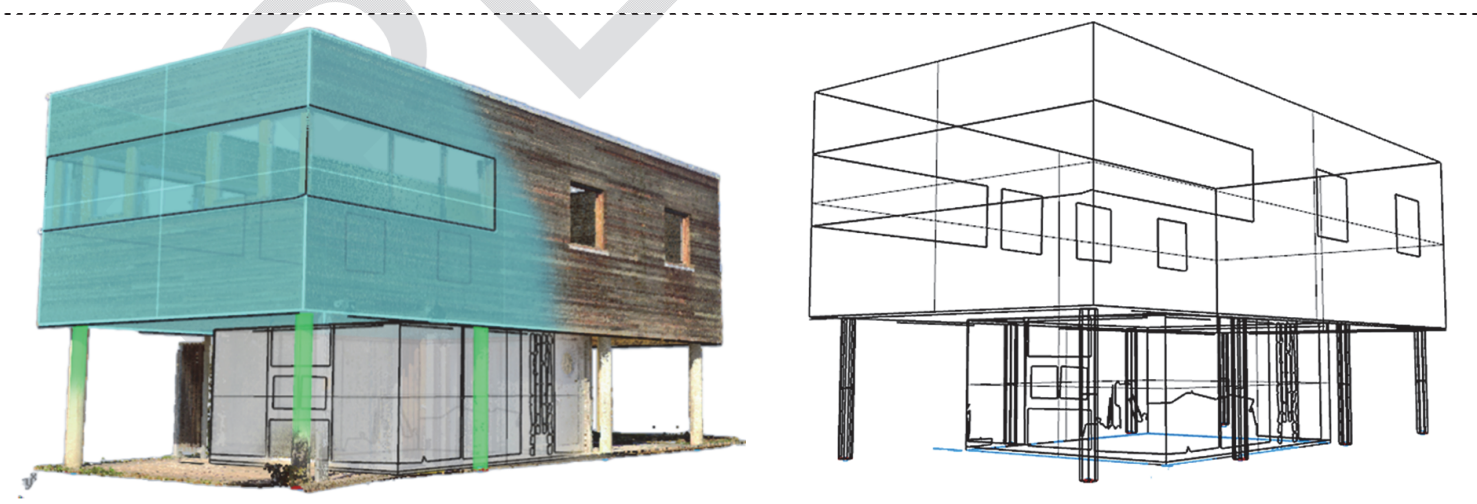

Figure 6. LEFT, illustration of the transformation of the PC_Set into a Ct_Set. RIGHT. 3D contour set (Ct_Set).

The general functioning of the RGH definition yielded most of the expected results. The most relevant are:

- Importing and downsampling PC_Sets for improving computing times.

- Splitting a PC_Set so non co-planar envelope segments are reconstructed separately, then regrouping into a single 3D Ct_Set. 
- Developing a strategy to avoid or bypass PC Set occlusions commonly found when scanning building envelopes.

- Proposing a Nurbs modelling workflow capable of reading building topology instead of limiting the solution to solving regular-parallel building topologies. This constitutes one of the main contributions of this work. It is achieved by implementing path extrusion and loft operations applied to horizontal envelope wall contours.

- A test using DURAARK'S GeomGym IFC plug-in for GH, allowed a digital workflow for transforming three-dimensional Ct_Sets into IFC compatible models suitable for enrichment via native BIM modelers such as Revit or Archicad.

\subsection{Accuracy}

As accuracy is an important aspect to consider when tackling facade retrofitting projects, the RGH definition accompanying the method proposed herein includes an accuracy measuring module. The module measures the minimum and maximum distances between a sample point in the PC_Set and a given wall footprint over a slicing plane. It calculates the average distance between both entities, the wall path and the PC_Set sample point, to provide the user with information regarding potential deviations.

At this point, the user can check whether facade planes (F-C_Planes) are:

a) Within a range inside the PC-Set slice (Pc_Set_a), so the user can verify whether reconstructed building faces will be properly placed on the corresponding XY plane.

b) Aligned to the PC Set segment being measured. This is possible by taking measures on different slicing planes, which in turn are placed at different relative heights. While the measured segment itself helps identify horizontal deviation, assessing the same criterion at different relative heights ensures the face Brep will follow any pre-existing vertical deviation the facade might have.

\section{How it works.}

The measurement module populates a wall path (Wp1) with a given number of sample points (SP_Set) which in turn are tested against the PC_Set segment to which Wp1 belongs (Figure 7). It uses a " $R G H$ closest point" [51] component to measure the distance between sample points and their closest point in the PC_Set (Figure 7, right). Figure 7 shows that a wall path (Wp1) is measured against a PC_Set segment (PC_Set_a) belonging to a given building facade $\left(B f_{-} 1\right)$.

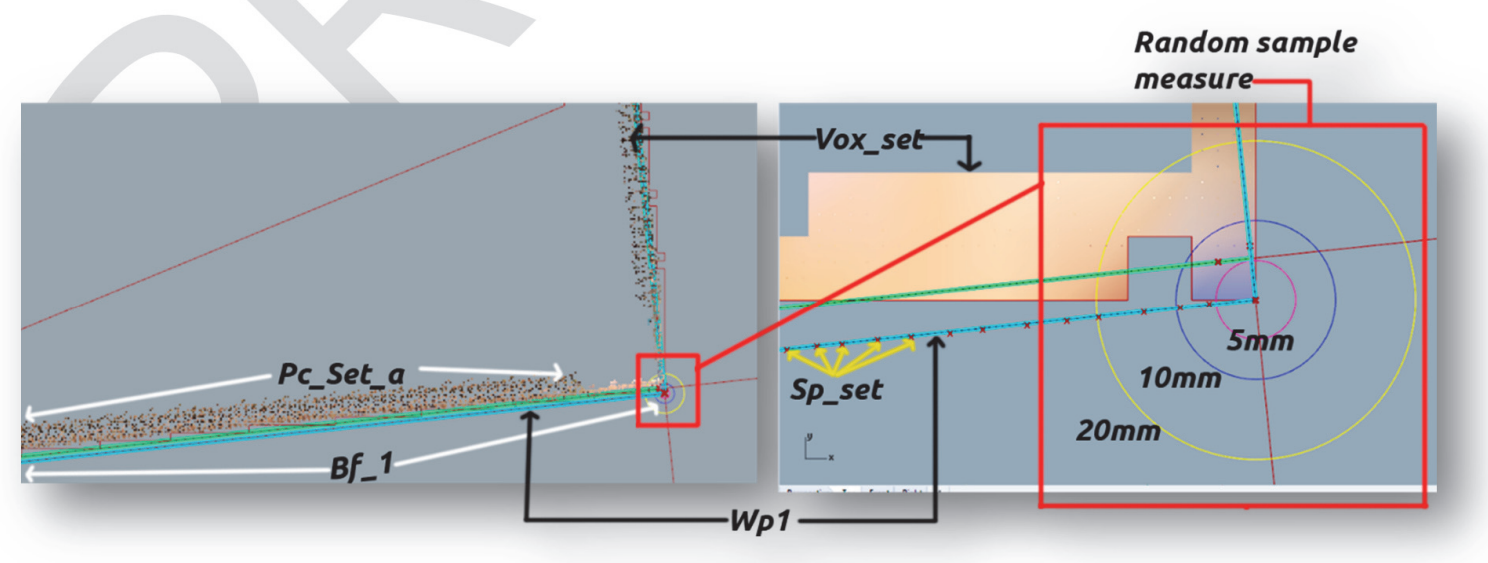

Figure 7. Accuracy test- LEFT, sample segment on a PC_Set slice. RIGHT. Reference distances around a random sample point for measuring. Measured points are contained within a voxel set from which wall paths are retrieved 
According to the graph in Figure 8, about 51 sample points are within $2.9 \mathrm{~mm}$ and $10 \mathrm{~mm}$ from their closest point along PC_Set_a. Only eight are in a range between 10 and $13.5 \mathrm{~mm}$ from their closest point.

This function's purpose is to provide data supporting accuracy metrics during the digital reconstruction process.

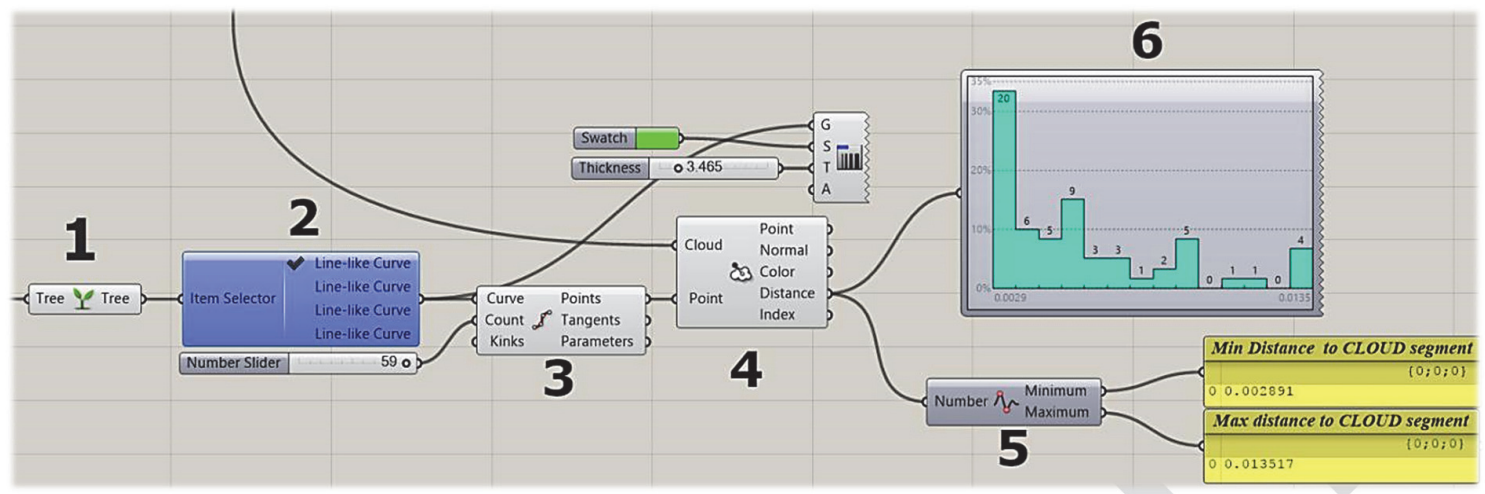

Figure 8. Accuracy measurement tool. 1 Wall footprint set (as tree); $\mathbf{2}$ Wall footprint selector; $\mathbf{3}$ sample point creator; 4 PC_Set closest point component; 5 and 6 Measurements.

\section{Conclusion and perspectives}

Though a work in progress, the proposed method along with its RGH definition have fulfilled the aims of this work so far. The definition can slice the PC_Set of a building envelope in order to obtain wall footprints that later help generate a Nurbs representation of the building's envelope. The same slicing method is used to retrieve window and door openings in the form of Ct_Sets that later help split the envelope's Brep. By running a series of operations, contours are regrouped in a manner that they represent defined element categories: Envelope walls, windows, doors, and even columns.

The final three-dimensional Ct_Set constitutes a valuable set of geometric data usable for facade retrofitting projects in which accuracy is crucial. A further step in exploiting the 3D Ct_Set showed that it also helps produce IFC files suitable for further BIM enrichment.

Upcoming development stages will aim to improve functions regarding topics such as:

- Occlusion. So far, the proposed method is able to tackle some occlusion cases, which means more testing is necessary in order to document more occlusion scenarios and propose strategies to solve them.

- Building topology. More work is envisaged to tackle not only complex building topologies and morphologies but also manifold facade layouts since the performed tests have proven to work well in relatively flat facades. This means, facade elements such as cornices, sills, casements, keystones, pilasters, and rails, among many others, are not yet treated. We acknowledge that the aforementioned remains a challenge in the digital reconstruction field, as cited works also lack capabilities for reconstructing such architectural items.

Nevertheless, and because of the use of parametric Nurbs modelling, the proposed method has potential for reconstructing freeform building geometries, which is an important limitation of RANSAC-based methods that depend on PC_Set fitting planes for retrieving building contours. 


\section{Acknowledgements}

This work was possible thanks to the funding of France's Grand East region, the "CPER FORBOIS" project and the project for "applied BIM-driven strategic and operational cloud services for facilities evolution - UBIK".

\section{References}

[1] European Commision. Energy performance of buildings directive. 2019; 8.

[2] THE EUROPEAN PARLIAMENT; THE COUNCIL OF THE EUROPEAN UNION. DIRECTIVE (EU) 2018/844 OF THE EUROPEAN PARLIAMENT AND OF THE COUNCIL of 30 May 2018. Official Journal of the European Union 2018; 17.

[3] Matarneh ST, Danso-Amoako M, Al-Bizri S, et al. Building information modeling for facilities management: A literature review and future research directions. J Build Eng 2019; 24: 100755.

[4] Hackel T, Wegner JD, Schindler K. Contour Detection in Unstructured 3D Point Clouds. 2016; 16101618.

[5] Cha H, Lee D. Framework Based on Building Information Modelling for Information Management by Linking Construction Documents to Design Objects. J Asian Archit Build Eng 2018; 17: 329-336.

[6] Khabazi Z. Generative Algorithms (using Grasshopper). morphogenesism, http://morphogenesism.com (2012).

[7] Wortmann T, Tunçer B. Differentiating parametric design: Digital workflows in contemporary architecture and construction. Des Stud 2017; 52: 173-197.

[8] Thomson C, Boehm J. Automatic Geometry Generation from Point Clouds for BIM. Remote Sens 2015; 7: 11753-11775.

[9] Tedeschi A. Parametric architecture with Grasshopper ${ }^{\circledR}$ : primer. Brienza, Italy: Le Penseur, 2011.

[10] Borodinecs A, Zemitis J, Dobelis M, et al. Modular retrofitting solution of buildings based on 3D scanning. Procedia Eng 2017; 205: 160-166.

[11] Barco Santa AF. Constraint-based design : two-dimensional insulating panels configuration. CGI - Centre Génie Industriel, http://www.theses.fr/2016EMAC0006/document.\%0A (2016).

[12] Gámez O, Bignon J-C, Duchanois G. Assisted Construction of Non-standard Wooden Walls and Envelope Structures by Parametric Modeling. In: Celani G., Sperling D. FJ (ed) Communications in Computer and Information Science. Sao Paulo: Springer, pp. 291-308.

[13] Heaton J, Parlikad AK, Schooling J. Design and development of BIM models to support operations and maintenance. Comput Ind 2019; 111: 172-186.

[14] Araszkiewicz K. Digital Technologies in Facility Management - The state of Practice and Research Challenges. Procedia Eng 2017; 196: 1034-1042.

[15] Bitelli G, Balletti C, Brumana R, et al. METRIC DOCUMENTATION OF CULTURAL HERITAGE: RESEARCH DIRECTIONS FROM THE ITALIAN GAMHER PROJECT. ISPRS - Int Arch Photogramm Remote Sens Spat Inf Sci 2017; XLII-2/W5: 83-90.

[16] Wang $Y$, Ma Y, Zhu A, et al. Accurate facade feature extraction method for buildings from threedimensional point cloud data considering structural information. ISPRS J Photogramm Remote Sens 2018; 139: 146-153.

[17] Xue F, Lu W, Chen K. Automatic Generation of Semantically Rich As-Built Building Information Models Using 2D Images: A Derivative-Free Optimization Approach. Comput Civ Infrastruct Eng 2018; 33: 926 942. 
[18] Tamke M, Zwierzycki M, Evers HL, et al. Tracking changes in buildings over time - Fully automated reconstruction and difference detection of 3D scan and BIM files. In: the 34th eCAADe Conference. 2016, pp. 643-651.

[19] Macher H, Landes T, Grussenmeyer P. From Point Clouds to Building Information Models: 3D SemiAutomatic Reconstruction of Indoors of Existing Buildings. Appl Sci 2017; 7: 1030.

[20] Previtali M, Scaioni M, Barazzetti L, et al. A flexible methodology for outdoor/indoor building reconstruction from occluded point clouds. ISPRS Ann Photogramm Remote Sens Spat Inf Sci 2014; II-3: 119-126.

[21] Jung J, Stachniss C, Ju S, et al. Automated 3D volumetric reconstruction of multiple-room building interiors for as-built BIM. Adv Eng Informatics 2018; 38: 811-825.

[22] Bennis A. Contribution à la reconstruction 3D de bâtiments à partir de nuage de points de scanner laser terrestre. $h t t p: / / w w w . t h e s e s . f r$, http://www.theses.fr/2015LORR0139 (2015, accessed 10 July 2019).

[23] Xue F, Lu W, Chen K, et al. From Semantic Segmentation to Semantic Registration: Derivative-Free Optimization-Based Approach for Automatic Generation of Semantically Rich As-Built Building Information Models from 3D Point Clouds. J Comput Civ Eng 2019; 33: 04019024.

[24] Adán A, Quintana B, Prieto SA, et al. Scan-to-BIM for 'secondary' building components. Adv Eng Informatics 2018; 37: 119-138.

[25] Kropp C, Koch C, König M. Interior construction state recognition with 4D BIM registered image sequences. Autom Constr 2018; 86: 11-32.

[26] Hinks T, Carr H, Truong-Hong L, et al. Point Cloud Data Conversion into Solid Models via Point-Based Voxelization. J Surv Eng 2013; 139: 72-83.

[27] Zolanvari SMI, Laefer DF, Natanzi AS. Three-dimensional building façade segmentation and opening area detection from point clouds. ISPRS J Photogramm Remote Sens 2018; 143: 134-149.

[28] Evers M, Wessel HL, Ochmann R; , et al. An Automated Approach to the Generation of Structured Building Information Models from Unstructured 3d Point Cloud Scans. I Proceedings of the IASS Annual Symposium, http://www.clearedge3d.com/ (2016, accessed 6 May 2020).

[29] Bassier M, Vergauwen M. Clustering of Wall Geometry from Unstructured Point Clouds Using Conditional Random Fields. Remote Sens 2019; 11: 1586.

[30] Laing R, Leon M, Isaacs J, et al. Scan to BIM: the development of a clear workflow for the incorporation of point clouds within a BIM environment. In: Building Information Modelling (BIM) in Design, Construction and Operations. WIT Press, 2015, pp. 279-289.

[31] Huhnt W. Reconstruction of edges in digital building models. Adv Eng Informatics 2018; 38: 474-487.

[32] BIMForum. Level of Development Specification, https://bimforum.org/wpcontent/uploads/2019/04/LOD-Spec-2019-Part-I-and-Guide-2019-04-29.pdf (2019).

[33] López F, Lerones P, Llamas J, et al. A Review of Heritage Building Information Modeling (H-BIM). Multimodal Technol Interact 2018; 2: 21.

[34] Barazzetti L, Banfi F, Brumana R, et al. Creation of Parametric BIM Objects from Point Clouds Using Nurbs. Photogramm Rec 2015; 30: 339-362.

[35] Issa R. Essential Mathematics for Computational Design. Robert McNeel \& AssociatesAssociates, https://www.rhino3d.com/download/rhino/6/essentialmathematics (2019).

[36] Barazzetti L. Parametric as-built model generation of complex shapes from point clouds. Adv Eng Informatics 2016; 30: 298-311.

[37] Dimitrov A, Gu R, Golparvar-Fard M. Non-Uniform B-Spline Surface Fitting from Unordered 3D Point Clouds for As-Built Modeling. Comput Civ Infrastruct Eng 2016; 31: 483-498. 
[38] Faro. FARO As-Built - Complete Modeling of Reality Capture Data | FARO Asia Pacific, https://www.faro.com/en-sg/products/construction-bim/faro-as-built/ (accessed 15 April 2020).

[39] Scan to Bim. Scan To BIM - Revit add-in for point cloud feature extraction, https://scantobim.xyz/ (accessed 15 April 2020).

[40] Scalypso. BIM Module - Scalypso, https://www.scalypso.com/en/bim-module/ (accessed 15 April 2020).

[41] Leica. Leica CloudWorx for Revit | Leica Geosystems, https://leica-geosystems.com/products/laserscanners/software/leica-cloudworx/leica-cloudworx-revit (accessed 15 April 2020).

[42] Janssen P, Stouffs R. Types of Parametric Modelling. In: Proceedings of the 20th International Conference of the Association for Computer-Aided Architectural Design Research in Asia (CAADRIA 2015) /. Daegu, South Korea, pp. 157-166.

[43] Modelab. The Grasshopper Primer. 3.3. Modelab, http://grasshopperprimer.com/en/ (2015).

[44] A Dimitrov MG-F. Robust NURBS Surface Fitting from Unorganized 3D Point Clouds for Infrastructure As-Built Modeling. In: Proceedings of the 31st International Conference of CIB W78, Orlando, Florida, USA, 23-25 June. Orlando, pp. 81-88.

[45] Grevit. Components | Grevit Documentation, https://grevit.gitbooks.io/grevitdocumentation/content/components.html (2020, accessed 25 August 2019).

[46] Mirtschin J. IFC Grasshopper - Geometry Gym, https://technical.geometrygym.com/rhinograsshopper/ifc/ifc-grasshopper (2019, accessed 20 July 2019).

[47] VisualArq. Grasshopper components - VisualARQ, https://www.visualarq.com/features/grasshoppercomponents/ (2020, accessed 18 March 2020).

[48] McNeel. Rhino to Revit and Back. McNeel Wiki 2020; 1.

[49] IAI International Council Limited. \{IFC2x\} \{Edition\} 3, http://www.buildingsmarttech.org/ifc/IFC2x4/alpha/html/ifcsharedbldgelements/lexical/ifcwall.htm (2006, accessed 18 April 2016).

[50] Building Smart International. IFC Schema Specifications - buildingSMART Technical, https://technical.buildingsmart.org/standards/ifc/ifc-schema-specifications/ (2017, accessed 8 November 2019).

[51] BesI PJ, McKay ND. A Method for Registration of 3-D Shapes. IEEE Trans Pattern Anal Mach Intell 1992; 14: 239-256. 